\title{
PPI schützen nicht vor dem Ösophaguskarzinom
}

Dass das Barrettsyndrom als Folge einer gastroösophagealen Refluxkrankheit das Risiko für ein distales Ösophaguskarzinom erhöht, ist allseits bekannt. Jetzt konnte in einer Studie gezeigt werden, dass die Einnahme von ASS bzw. NSAR vor einem solchen Karzinom schützt, jedoch nicht die Gabe eines PPI.

- Analysiert wurden die Daten von Patienten mit einem Adenokarzinom des Ösophagus (365 Patienten), einem Karzinom des gastroösophagealen Übergangs ( 426 PatiPräkanzerose enten) und einem PlattenepithelkarBarrettösophagus zinom (303 Patienten). Die Daten wurden mit einem Kontrollkollektiv aus einem Bevölkerungsregister verglichen.

Bei Patienten mit häufiger Refluxsymptomatik (mindestens $1 \mathrm{x} /$ Woche) stieg das Risiko für ein Adenokarzinom des Ösophagus um das 6,4-Fache, das Risiko für ein Karzinom im Bereich des gastroösophagealen Übergangs um das 4,6Fache und für ein Plattenepithelkarzinom um das 2,2-Fache. Bei Rauchern war das Risiko für alle diese drei Tumorarten signifikant erhöht im Vergleich zu Nichtrauchern. Dagegen war das Karzinomrisiko bei Patienten, die regelmäßig ASS oder NSAR einnahmen, signifikant reduziert, nicht jedoch bei regelmäßiger Einnahme von Säuresekretionshemmern.

\section{Kommentar}

Dass die regelmäßige Einnahme von Säuresekretionshemmern das Ösophaguskarzinomrisiko nicht reduziert, ist überraschend. Bisher glaubte man, durch eine frühzeitige intensive Therapie der Refluxkrankheit auch die Entstehung eines Barrettsyndroms und somit die Entstehung eines Malignoms verhindern zu können. Es wird noch weiterer Studien bedürfen, um definitiv eine über die symptomatische Wirkung hinausgehende Effektivität der PPI im Hinblick auf die Verhinderung eines Ösophaguskarzinoms ausschließen zu können. Ansonsten wäre die Gabe eines solchen Medikamentes nur im Hinblick auf die Symptomkontrolle sinnvoll.

P. Stiefelhagen .

- N. Pandeya et al.

Gastroesophageal reflux symptoms and the risks of esophageal cancer: Are the effects modified by smoking. NSAIDs or acid suppressants? Gut 59 (2010) 31-38 